\title{
Fingolimod in pediatric-onset multiple sclerosis
}

\author{
Mauro Zaffaroni ${ }^{1}$
}

Received: 27 April 2021 / Accepted: 28 April 2021 / Published online: 4 May 2021

(C) Fondazione Società Italiana di Neurologia 2021

Multiple sclerosis (MS) is a chronic inflammatory immunemediated disease in which demyelination and degeneration of nerve fibers in the central nervous system disrupt the transmission of nerve signals from the brain and spinal cord to other parts of the body. The symptoms of MS vary among patients, in their severity and duration, and can include pain, fatigue, sensory disturbances, vision problems, muscle weakness and spasticity, sphincter dysfunction, and impaired coordination and cognition $[1,2]$.

Although MS more commonly occurs in young adults aged between 20 and 40 years [3], approximately $2-10 \%$ of all patients with MS will experience disease onset during childhood or adolescence $[4,5]$. Pediatric-onset MS, defined as the onset of MS before 18 years of age, occurs in fewer than 1 in 100,000 children per year; the medium age at onset is 15 years and $>90 \%$ of patients are $\geq 10$ years of age [6-8].

Pediatric-onset MS typically follows a relapsing-remitting course, in which patients experience relapses of MS followed by periods of stability, with a twofold to threefold higher rate of relapse than in adults $[9,10]$. As a consequence, children with MS tend to reach disability milestones at a younger age than adults with MS [8].

In pediatric MS, we are as close as possible to the biological onset of the disease, and therefore, treatment received at this early stage is particularly important to determine the longterm outcome of the patient.

For adults with relapsing-remitting MS, several diseasemodifying therapies are available as traditional first-line treatments, including interferon $\beta$ and glatiramer acetate; however, their efficacy and safety in the pediatric MS population remains to be evaluated in prospective, randomized clinical trials [11]. Notably, the optimal treatment strategy for pediatriconset MS is currently unknown, and a considerable proportion

Mauro Zaffaroni

mauro.zaffaroni@asst-valleolona.it

1 Multiple Sclerosis Centre, Hospital of Gallarate, ASST della Valle Olona, Via Pastori 4, 21013 Gallarate, Italy of pediatric patients are undertreated. First-line treatments are often suboptimal due to the high level of disease activity in patients with pediatric-onset MS, and switching to alternative treatment strategies may be required. In a recent analysis of 288 newly diagnosed pediatric MS patients, approximately two-thirds of patients were not treated within the first year of diagnosis [12]. Interferons and glatiramer acetate were the most commonly initiated treatments, although almost onethird of patients experienced early treatment failure and discontinued or switched within 6 months of treatment initiation.

Natalizumab is a potential treatment option for pediatric MS patients with an aggressive course or those with an inadequate response to first-line agents [13]; however, it is associated with an increased risk of progressive multifocal leukoencephalopathy (PML) and patients must be monitored closely [14].

Fingolimod (Gilenya®, Novartis), which acts on sphingosine-1-phosphate receptors by modulating lymphocyte trafficking and chemotactic responses [15], was the first disease-modifying therapy to be approved, in 2018, for the treatment of MS in pediatric patients by the United States Food and Drug Administration and the European Medicines Agency [16, 17]. Approval was based on the results of the randomized, phase 3 trial PARADIGMS. PARADIGMS (published in 2018) demonstrated the superior efficacy of oral fingolimod $0.5 \mathrm{mg}$ per day to intramuscular interferon $\beta-1 \mathrm{a}$ $30 \mu \mathrm{g}$ per week in 215 patients with relapsing MS who were aged 10 to 17 years [18]. Over the 2-year treatment period, significantly lower rates of relapse and of new or newly enlarged lesions on magnetic resonance imaging (MRI) were identified in patients treated with fingolimod compared with interferon $\beta$-1a.

In pediatric patients (aged 10 years and over), the recommended daily oral dose of fingolimod is dependent on body weight; $0.25 \mathrm{mg}$ orally once daily for patients $\leq 40 \mathrm{~kg}$ and $0.5 \mathrm{mg}$ orally once-daily for patients $>40 \mathrm{~kg}[16,17]$.

To achieve optimal outcomes, early diagnosis and appropriate treatment of pediatric MS patients is imperative. When 
to initiate therapy and the choice of first-line treatment are widely discussed topics in the management of these patients. In this supplemental issue of Neurological Sciences, medical professionals based in Italy describe their experiences of the effectiveness and safety of fingolimod from the pediatric MS compassionate use program.

Several cases reported here highlight the effectiveness and safety of fingolimod in children with active MS and an inadequate response to oral or injected first-line agents. Notably, fingolimod was efficacious as a second-line treatment in a 14year-old female diagnosed with pediatric-onset MS who had initially undergone treatment with interferon $\beta$-1a but experienced radiologic progression within 3 months of initiating treatment, and clinical relapse after 9 months. In this case report, described by Amidei and colleagues, the patient was switched to second-line treatment with fingolimod due to the high clinical and radiological disease activity she had experienced despite 12 months of treatment with interferon $\beta-1$ a. Fingolimod reduced the accumulation of lesions on MRI, with only two new minor brain lesions after 5 months of treatment. The patient was asymptomatic at 11 months after starting fingolimod and has subsequently experienced clinically and radiologically stability. Clinically stable disease has been maintained over the 2-year treatment period, with no treatment-related adverse events reported to date.

Zanetta and colleagues describe two cases of children with relapsing-remitting MS who experienced marked clinical benefit from second-line treatment with fingolimod. For both case reports, fingolimod treatment was well-tolerated, and neither patient has experienced any relevant clinical and/or hematologic adverse events. In the first case report, a female diagnosed with MS at 13 years of age initiated treatment with interferon $\beta$-1a but experienced subsequent clinical relapse, characterized mainly by increased gait disturbances and the presence of neuroradiologic disease activity, shown by an increase of lesional load on brain MRI. The patient was switched to natalizumab; however, her risk of developing PML increased after 20 infusions, and, for safety reasons, she discontinued natalizumab and initiated fingolimod $0.5 \mathrm{mg}$ daily. The patient has received fingolimod for 29 months and has experienced neither clinical relapse nor the presence of neuroradiologic disease activity.

The second case report from Zanetta and colleagues describes a 15-year-old female diagnosed with MS who had responded inadequately to first-line treatment with dimethyl fumarate; neuroradiologic disease activity was identified by follow-up brain MRI after 1 month of treatment, with three new brain lesions identified after 6 months. After switching to fingolimod $0.5 \mathrm{mg}$ daily, this patient experienced both clinical and neuroradiologic disease stabilization. The patient has received fingolimod for 33 months and remains free from disease activity.
The case report by Immovilli and colleagues also demonstrates the effectiveness of second-line treatment with fingolimod after interferon $\beta$-1a treatment failure in an adolescent female with MS. In this case report, the patient was diagnosed with MS at the age of 13 years and was initially treated with interferon $\beta-1 \mathrm{a}$ for 2 years but relapsed, with MRI showing a new cerebral enhancing lesion and a new cervical lesion in the left dorsal column segments C5 and C6. The patient was switched to natalizumab, which was not welltolerated after only two infusions and was subsequently discontinued. Fingolimod $0.5 \mathrm{mg} /$ day orally was initiated (the patient's body weight was $67 \mathrm{~kg}$ ). Brain and cervical MRI after 1.5 years of active treatment showed no new, enlarging, or enhancing lesions compared with the rebaseline MRI, which was obtained after 7 months of active treatment. At her 2.5-year follow-up visit, the patient was still receiving active fingolimod treatment and has shown no clinical or radiological activity, giving the patient a status of no evidence of disease activity (NEDA 3), which is predictive of no disease progression at 7 years after initiating fingolimod treatment [19]. Importantly, fingolimod has been well-tolerated and has not interfered with daily-life activities.

Valeriani and colleagues present two case studies in pediatric-onset MS and discuss the benefits of early switching to fingolimod following relapse on first-line treatment. A suboptimal response to first-line glatiramer acetate in a 16-year old female with a confirmed diagnosis of severe MS prompted escalation to fingolimod $0.50 \mathrm{mg} /$ day orally as second-line therapy. Notably, the patient did not have any symptoms while receiving glatiramer acetate; however, MRI of the brain, brain stem, and cervical spinal cord identified new lesions after 1 year of treatment. An MRI scan after 1 year of fingolimod showed no further disease progression and neurological examination of the patient was normal (Expanded Disability Status Scale $($ EDSS $)=0)$. The patient has not experienced any adverse events and has continued with fingolimod treatment. The second case report describes a 17year-old female with MS who was initially treated with interferon $\beta$-1a for 2 years but then switched to second-line treatment with fingolimod $(0.50 \mathrm{mg} /$ day orally) when a follow-up MRI showed a new brain lesion. A follow-up MRI scan after 1 year and 5 months since starting fingolimod showed no signs of disease progression, and neurological examination was unremarkable (EDSS $=0$ ). The patient has continued fingolimod treatment, which has been well-tolerated.

Together, these six case reports highlight the need for prompt escalation to second-line therapies, such as fingolimod, following relapse to first-line treatment. This is particularly important for patients with pediatric-onset MS as appropriate treatment is imperative to optimize outcomes.

Due to the highly active and aggressive course of pediatriconset MS, early initiation of effective disease-modifying drugs is imperative to prevent the accumulation of irreversible 
disability and to preserve both physical and cognitive functioning [20]. However, the optimal treatment approach is unclear and discussions are ongoing regarding the effectiveness of treatment escalation or the early initiation of a highly active therapy, such as fingolimod, as a first-line treatment. In the case report by Capobianco, the higher-efficacy disease-modifying therapy, fingolimod, was used as a first-line treatment in a female who was diagnosed with highly active relapsingremitting MS at the age of 14 years. Fingolimod, which was initiated within 1 year from the first recorded symptom, has stabilized the disease course and no disease progression has been observed since starting treatment. Notably, the patient's EDSS score improved from 6.0 at first presentation to 2.0 after 1 year of treatment, clinical relapses have been absent, and MRIs performed after 3 and 12 months of fingolimod treatment have shown no evidence of disease activity.

Fingolimod was also used successfully as a first-line treatment in a 12-year-old male with severe MS in a case study presented by Valeriani and colleagues. The patient, who was initially admitted with optic neuritis in the left eye, with an EDSS score of 2, was diagnosed with aggressive MS following an MRI scan showing evidence of numerous infratentorial and spinal lesions. First-line fingolimod $0.25 \mathrm{mg}$ /day orally was initiated within 3 months of diagnosis and has been welltolerated throughout the treatment period. Subsequent MRI scans after 6 and 11 months of treatment showed no sign of disease progression. Neurological examination was normal at the last follow-up visit $($ EDSS $=0)$ and fingolimod treatment continues.

The successful outcomes of both case reports add further support to the use of first-line fingolimod as a highly active treatment option in pediatric patients with aggressive MS.

Data are currently lacking on the long-term (i.e., $>2$ years) safety and efficacy of fingolimod in children with MS. Borriello and Pozzilli present two cases of children with pediatric-onset MS who achieved sustained clinical benefit from treatment with fingolimod for more than 2 years. The first case report describes an 11-year-old male with relapsingremitting MS who was a participant in the PARADIGMS study and was treatment-naïve at the time of fingolimod initiation. This patient has since received prolonged treatment with first-line fingolimod for 5 years and has remained clinically stable throughout, with no adverse events reported. Annual MRI scans have shown no new lesions or radiological lesion progression, and there has been no change in neurological status since diagnosis (EDSS $=2$ ).

The second case describes a 12-year-old female with relapsing-remitting MS who was switched to fingolimod 2 years after being diagnosed with pediatric-onset MS and after experiencing two relapses within 8-months of starting interferon $\beta$-1a. Since starting fingolimod, the patient has had no relapses or new or active MRI lesions in more than 3 years of treatment with fingolimod, her EDSS score has decreased from 3 to 2, and she has remained clinically stable. For both patients, treatment with fingolimod was well-tolerated.

These case reports suggest that, in pediatric patients with MS, prolonged treatment (i.e., >2 years) with fingolimod can be used safely as first-line or second-line therapy, with patients experiencing clinical benefits such as the prevention of relapse and lesion load accrual, achieving/maintaining clinical stability, and delayed disease progression.

Administration of the first fingolimod dose has been associated with a dose-dependent, transient asymptomatic bradycardia within the first $6 \mathrm{~h}$ post-dose in a small number of predisposed patients [21-23]. Hence, it is vital for electrocardiogram (ECG) monitoring of a patient's heart rate during the initial $6 \mathrm{~h}$ after the first administration of fingolimod. Petruzzo and Lanzillo describe the case of a 17-year-old female with relapsing-remitting MS who experienced asymptomatic bradycardia with a Mobitz type I second-degree atrioventricular block at approximately $5.5 \mathrm{~h}$ after receiving her first dose of fingolimod. This patient had experienced persistent radiological activity despite 3 years of treatment with high-dose interferon $\beta-1 \mathrm{a}$ and was subsequently switched to fingolimod $0.5 \mathrm{mg}$ orally. No cardiac symptoms were observed during pre-treatment assessments, cardiovascular examination was normal, 12-lead ECG was unremarkable (heart rate of 76 beats per minute (bpm)), and there was no significant cardiovascular disease in her history. At approximately $5.5 \mathrm{~h}$ after administration of the first dose of fingolimod, a second-degree atrioventricular block (Mobitz type I) was identified by ECG. The patient's heart rate was $54 \mathrm{bpm}$ at nadir, which continued until the end of the 6-h monitoring period requiring extended overnight monitoring during which the patient remained asymptomatic and treatment was not required. Her ECG reverted to sinus rhythm with a normal PR interval at approximately $12 \mathrm{~h}$ post the first dose of fingolimod, and no adverse cardiac events were reported at the 1-month and 3-month follow-up visits (heart rate of 72-76 bpm). This case report highlights the importance of heart rate monitoring during the first $6 \mathrm{~h}$ post-administration of the first fingolimod dose. Despite a generally reassuring safety profile of fingolimod in pediatric MS patients, there is a real need for more real-life data on adverse events of fingolimod in pediatric MS patients in order to better understand the risks involved during treatment.

In conclusion, fingolimod appears to be an effective and safe therapeutic agent for the management of the highly active and aggressive disease course of pediatric-onset MS. This is further supported by its oral route of administration, effectiveness, and good tolerability.

The case reports presented here highlight the effectiveness and safety of fingolimod as a first-line or second-line treatment option in pediatric-onset MS in daily clinical practice. Although data to guide treatment choice are currently limited, the experiences of fingolimod from cases within the pediatric MS compassionate use program will ultimately further our 
understanding of the role of fingolimod in the pediatric MS population.

Acknowledgements I would like to thank Melanie Gatt (PhD), an independent medical writer, who prepared the first draft of this article on behalf of Springer Healthcare Communications. This medical writing assistance was funded by Novartis.

\section{Declarations}

Conflict of interest MZ received travel support, compensation for serving on scientific advisory board, or speaker's fees from Almirall, Biogen, Merck Serono, Novartis, and Sanofi-Genzyme.

Ethical approval None.

Informed consent None.

\section{References}

1. Goldenberg MM (2012) Multiple sclerosis review. PT 37:175-184

2. Reich DS, Lucchinetti CF, Calabresi PA (2018) Multiple sclerosis. N Engl J Med 378:169-180. https://doi.org/10.1056/ NEJMra1401483

3. Confavreux C, Aimard G, Devic M (1980) Course and prognosis of multiple sclerosis assessed by the computerized data processing of 349 patients. Brain 103:281-300. https://doi.org/10.1093/brain/ 103.2.281

4. Alroughani R, Boyko A (2018) Pediatric multiple sclerosis: a review. BMC Neurol 18:27. https://doi.org/10.1186/s12883-0181026-3

5. Waldman A, Ghezzi A, Bar-Or A, Mikaeloff Y, Tardieu M, Banwell B (2014) Multiple sclerosis in children: an update on clinical diagnosis, therapeutic strategies, and research. Lancet Neurol 13:936-948. https://doi.org/10.1016/S1474-4422(14)70093-6

6. Absoud M, Lim MJ, Chong WK, De Goede CG, Foster K, Gunny $R$ et al (2013) Paediatric acquired demyelinating syndromes: incidence, clinical and magnetic resonance imaging features. Mult Scler 19:76-86. https://doi.org/10.1177/1352458512445944

7. Banwell B, Kennedy J, Sadovnick D, Arnold DL, Magalhaes S, Wambera K, Connolly MB, Yager J, Mah JK, Shah N, Sebire G, Meaney B, Dilenge ME, Lortie A, Whiting S, Doja A, Levin S, MacDonald EA, Meek D, Wood E, Lowry N, Buckley D, Yim C, Awuku M, Guimond C, Cooper P, Grand'Maison F, Baird JB, Bhan $\mathrm{V}$, Bar-Or A (2009) Incidence of acquired demyelination of the CNS in Canadian children. Neurology 72:232-239. https://doi. org/10.1212/01.wnl.0000339482.84392.bd

8. Renoux C, Vukusic S, Mikaeloff Y, Edan G, Clanet M, Dubois B, Debouverie M, Brochet B, Lebrun-Frenay C, Pelletier J, Moreau T, Lubetzki C, Vermersch P, Roullet E, Magy L, Tardieu M, Suissa S, Confavreux C (2007) Natural history of multiple sclerosis with childhood onset. N Engl J Med 356:2603-2613. https://doi.org/ 10.1056/NEJMoa067597

9. Benson LA, Healy BC, Gorman MP, Baruch NF, Gholipour T, Musallam A, Chitnis T (2014) Elevated relapse rates in pediatric compared to adult MS persist for at least 6 years. Mult Scler Relat Disord 3:186-193. https://doi.org/10.1016/j.msard.2013.06.004
10. Gorman MP, Healy BC, Polgar-Turcsanyi M, Chitnis T (2009) Increased relapse rate in pediatric-onset compared with adultonset multiple sclerosis. Arch Neurol 66:54-59. https://doi.org/10. 1001/archneurol.2008.505

11. Ghezzi A, Amato MP, Makhani N, Shreiner T, Gartner J, Tenembaum S (2016) Pediatric multiple sclerosis: conventional first-line treatment and general management. Neurology 87:S97S102. https://doi.org/10.1212/WNL.0000000000002823

12. Benjamin G, Kolodny S, Wang M, Deshpande C (2020) Utilization and treatment patterns of disease-modifying therapy among pediatric patients with multiple sclerosis in the United States. Int J MS Care. https://doi.org/10.7224/1537-2073.2019-095

13. Chitnis T, Tenembaum S, Banwell B, Krupp L, Pohl D, Rostasy K, Yeh EA, Bykova O, Wassmer E, Tardieu M, Kornberg A, Ghezzi A, and for the International Pediatric Multiple Sclerosis Study Group (2012) Consensus statement: evaluation of new and existing therapeutics for pediatric multiple sclerosis. Mult Scler 18:116127. https://doi.org/10.1177/1352458511430704

14. Rensel M (2019) Long-term treatment strategies of pediatric multiple sclerosis, including the use of disease modifying therapies. Children (Basel) 6:73. https://doi.org/10.3390/children6060073

15. Brinkmann V, Davis MD, Heise CE, Albert R, Cottens S, Hof R, Bruns C, Prieschl E, Baumruker T, Hiestand P, Foster CA, Zollinger M, Lynch KR (2002) The immune modulator FTY720 targets sphingosine 1-phosphate receptors. J Biol Chem 277: 21453-21457. https://doi.org/10.1074/jbc.C200176200

16. European Medicines Agency Glienya (fingolimod) [summary of product characteristics]. https:/www.ema.europa.eu/en/medicines/ human/EPAR/gilenya. Accessed April 7, 2020

17. U.S. Food and Drug Administration Gilenya, (2019) (fingolimod). Prescribing information. Revised: 12/2019. https://www.novartis. us/sites/www.novartis.us/files/gilenya.pdf. Accessed 10 July 2020

18. Chitnis T, Arnold DL, Banwell B, Bruck W, Ghezzi A, Giovannoni $\mathrm{G}$ et al (2018) Trial of fingolimod versus interferon beta-1a in pediatric multiple sclerosis. N Engl J Med 379:1017-1027. https://doi. org/10.1056/NEJMoa1800149

19. Rotstein DL, Healy BC, Malik MT, Chitnis T, Weiner HL (2015) Evaluation of no evidence of disease activity in a 7-year longitudinal multiple sclerosis cohort. JAMA Neurol 72:152-158. https:// doi.org/10.1001/jamaneurol.2014.3537

20. Narula S, Hopkins SE, Banwell B (2015) Treatment of pediatric multiple sclerosis. Curr Treat Options Neurol 17:336. https://doi. org/10.1007/s11940-014-0336-Z

21. Cohen JA, Barkhof F, Comi G, Hartung HP, Khatri BO, Montalban X, Pelletier J, Capra R, Gallo P, Izquierdo G, Tiel-Wilck K, de Vera A, Jin J, Stites T, Wu S, Aradhye S, Kappos L (2010) Oral fingolimod or intramuscular interferon for relapsing multiple sclerosis. N Engl J Med 362:402-415. https://doi.org/10.1056/ NEJMoa0907839

22. Di Marco J (2012) Fingolimod treatment initiation experience: cardiac and Holter electrocardiogram findings from three phase 3 studies. Mult Scler 18(Suppl 4):55-227. https://doi.org/10.1177/ 1352458512459019

23. Kappos L, Radue EW, O'Connor P, Polman C, Hohlfeld R, Calabresi P et al (2010) A placebo-controlled trial of oral fingolimod in relapsing multiple sclerosis. N Engl J Med 362: 387-401. https://doi.org/10.1056/NEJMoa0909494

Publisher's note Springer Nature remains neutral with regard to jurisdictional claims in published maps and institutional affiliations. 